\title{
Observation of collective effects in the multiphoton ionization of atomic deuterium
}

\author{
P. Bowe, ${ }^{1, *}$ F. Giammanco, ${ }^{2}$ R. W. O’Neill, ${ }^{1}$ P. J. M. van der Burgt, ${ }^{1}$ and J. A. Slevin ${ }^{1}$ \\ ${ }^{1}$ Department of Experimental Physics, National University of Ireland, Maynooth, Maynooth, County Kildare, Ireland \\ ${ }^{2}$ Istituto Nazionale di Fisica della Materia e Dipartimento di Fisica, Università di Pisa, Piazza Torricelli 2, 56126 Pisa, Italy
}

(Received 20 October 1997)

\begin{abstract}
The yields and time-of-flight spectra for ions produced in three-photon ionization of atomic deuterium have been investigated as a function of laser intensity from 0.4 to $200 \mathrm{MW} / \mathrm{cm}^{2}$. A pulsed laser beam of $243 \mathrm{~nm}$ is used, so that the photoionization is resonant with the metastable $2 s$ state of deuterium. The production of resonantly excited $2 s$ atoms is measured by detecting the quenched Lyman- $\alpha$ radiation at time intervals both during and after the laser pulse. The near degeneracy of the $2 s-2 p$ state manifold provides a particularly sensitive way to monitor the presence of any electric field in the interaction volume. As the laser intensity is increased, we observe the effects of a collective field arising from the electron-ion plasma produced by the laser pulse. We present the results of a theoretical model where the collective motion of the ions and the electrons is analyzed in terms of the fluid approximation. The essential features of the experimental observations including ion yields, ion time-of-flight spectra, and metastable production versus time, are well described by the theory. [S1050-2947(98)06508-1]
\end{abstract}

PACS number(s): $32.80 . \mathrm{Rm}, 32.80 . \mathrm{Wr}, 52.25 . \mathrm{Wz}, 52.50 . \mathrm{Jm}$

\section{INTRODUCTION}

Multiphoton ionization of atoms, resonant as well as nonresonant, has been the focus of much attention over the last three decades from experimentalists and theoreticians alike. Experiments on multiphoton ionization of atoms are usually performed by crossing a pulsed laser beam with a beam of atoms inside a vacuum chamber. Laser intensities have increased from $10^{7} \mathrm{~W} / \mathrm{cm}^{2}$ for ns lasers to $10^{18} \mathrm{~W} / \mathrm{cm}^{2}$ for lasers with pulse lengths of several femtoseconds. As higher laser powers have become available the perturbing effect that the electric field of the laser has on the target atom and hence the multiphoton interaction has been the focus of much theoretical effort. Experimental observations of either electron or ion yields or both have provided very detailed information on the dynamics of the multiphoton process [1-4].

An inevitable consequence of any ionization process is the creation of electron-ion pairs. In experimental situations where intense lasers and large atom densities are employed, the density of ions and electrons can be high enough to create a plasma where the ion and electron motions are significantly perturbed by the coupling produced by the mutual electric fields of the charged particles. As a consequence, experimentally measured quantities such as electron and ion yields and electron and ion time-of-flight spectra are affected by the interplay of two physical processes: (i) the multiphoton ionization of the atoms, and (ii) the collective effects of the plasma.

Collective effects have been studied in detail by Giammanco [5,6], Arimondo et al. [7], and Giammanco and Arimondo [8], who carried out experiments on the three-photon resonant ionization of sodium. They used a dye laser in the 5390-6000- $\AA$ region and employed different excitation schemes, one of which was a two-photon process resonant

\footnotetext{
*Present address: Institute of Physics and Astronomy, Aarhus University, DK-8000 Aarhus C, Denmark.
}

with the $4^{2} D$ state. The relevance of collective fields has been recognized in a few experiments involving secondharmonic generation in atomic vapors. One method of producing second-harmonic generation involves application of an external static electric field. In the experiments of Mossberg et al. [9], Miyazaki et al. [10,11], and Marmet et al. [12] second-harmonic generation was observed in the absence of external fields and attributed to a charge-separation field due to the ions and electrons produced by the photoionization.

Despite the work on sodium by Giammanco and coworkers [5-8], little experimental work to date has been specifically devoted to the analysis of collective interactions and their influence on experimental measurements. In this paper, we present measurements of the three-photon ionization of atomic deuterium at $243 \mathrm{~nm}$. This system presents an ideal situation for the study of collective effects for two reasons. First, the deuterium atom is one of the lightest in the periodic table and therefore deuterium ions are very susceptible to the collective field. Second, since the $2 s$ state is nearly degenerate with the $2 p$ state and is quenched in relatively weak electric fields (of a few $100 \mathrm{~V} / \mathrm{cm}$ ) the resonant multiphoton ionization process in deuterium is very susceptible to Stark mixing of the $2 s$ and $2 p$ states in the collective field. Observation of the quenched Lyman- $\alpha$ radiation provides a powerful tool for detecting and investigating quantitatively the role of collective effects. By varying the laser intensity we have control over the number of ions and electrons created in the interaction region and hence the strength of the collective field.

In the present experiment we cross a pulsed laser beam with an atomic deuterium beam. A pulsed electric field applied in the interaction region simultaneously extracts the ions and quenches the metastable $2 s$ atoms, allowing both species to be detected. This external field can be applied synchronously with the laser pulse or delayed for several ns after the laser is switched off. Since the Lyman- $\alpha$ emission depends on $2 s-2 p$ mixing induced by an electric field, the 


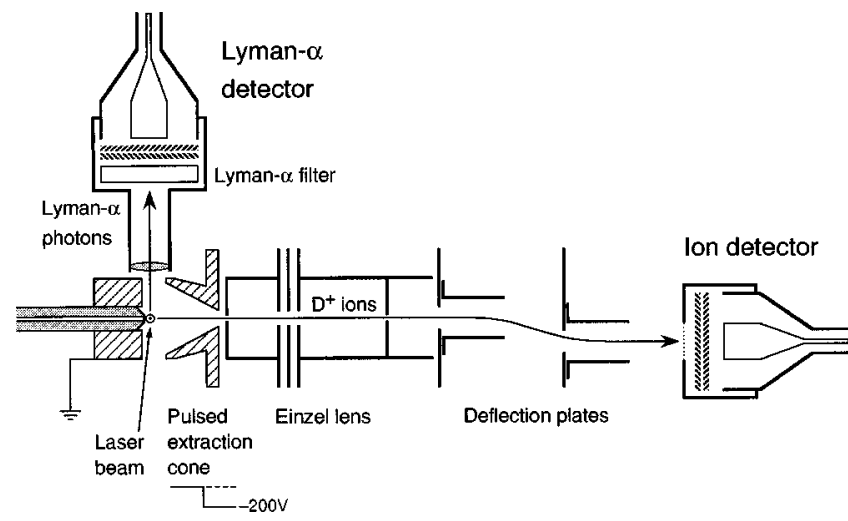

FIG. 1. Schematic overview of the interaction region, the ion optics, and the ion and Lyman- $\alpha$ detectors.

Lyman- $\alpha$ signal as a function of time is expected to be different depending on whether the external field is switched on during or after the laser pulse. The collective field introduces an additional quenching, which acts in a different way in both cases. When the external electric field is on during the laser pulse, it can be expected to modify the intermediate $2 s$ density and hence affect the production phase of the ionization process. Similarly, any collective field present will also modulate the ion production rate.

The experiment we report here is thus a rich mixture of atomic and plasma physics. Ionization is produced in an intense pulsed laser field where the laser photons are resonant with an intermediate state whose lifetime is strongly influenced by the presence of Stark fields, generated internally by collective plasma effects or externally by an applied electric field, or both. In each case the experimental signals are strongly dependent on the laser intensity, which sets directly the signature for all of the internal field-induced phenomena. The present experiment therefore presents an opportunity to observe a multiphoton ionization process in a fundamental atomic system where collective effects can be significant in the production phase because of the near degeneracy of the hydrogenic $2 s$ and $2 p$ states and where the quenched Lyman- $\alpha$ signal provides a unique opportunity for observing the influence of these fields in the ionization process.

In Sec. II of this paper we present a brief description of the experimental apparatus and techniques. In Sec. III we develop a theoretical model for the collective effects in the multiphoton ionization of deuterium. This model is based on the Boltzmann transport equations taking into account collective effects and the effects of the $2 s-2 p$ coupling both during and after the laser pulse. In Sec. IV we present our experimental data and compare the results with the theoretical model. A summary and conclusions are presented in Sec. V.

\section{DESCRIPTION OF APPARATUS AND EXPERIMENTAL TECHNIQUE}

The experimental setup is shown schematically in Fig. 1. Molecular deuterium is dissociated in a radio-frequency discharge and effuses through a 1-mm capillary into the interaction region realizing an atomic density of the order of $5 \times 10^{11}$ atoms $/ \mathrm{cm}^{3}$. In order to minimize the effects of Doppler broadening the laser beam intersects the atomic beam at right angles. We use the second-harmonic output of an excimer-pumped dye laser (Lambda Physik LEXTRA+LPD3002), tuned to the $1 s-2 s$ two-photon resonance at $243 \mathrm{~nm}$. The maximum pulse energy available is 1.8 $\mathrm{mJ}$. The temporal shape of the beam is approximately Gaussian with a pulse width of $13.5 \mathrm{~ns}$ full width at half maximum. The bandwidth of the laser at $243 \mathrm{~nm}$ is $0.36 \mathrm{~cm}^{-1}(10 \mathrm{GHz})$. The laser intensity is recorded on a shot-to-shot basis by an ultraviolet (UV) sensitive photodiode and calibrated with a separate measurement of the absolute energy using a pyroelectric detector. The output of the photodiode is fed into an analog-to-digital converter located in a personal computer.

Two photons at $243 \mathrm{~nm}$ excite the ground-state atoms to the $2 s$ level and the absorption of a further photon ionizes the atom. Two products of this interaction are detected viz., the residual ions from the ionization process and the Lyman- $\alpha$ radiation produced by quenching the metastable $2 s$ atoms. The ions are extracted by a pulsed electric field of $480 \mathrm{~V} / \mathrm{cm}$ applied in the interaction region. The extracted ions are focused by an einzel lens and pass a short drift region and a dual set of deflection plates, before hitting a pair of stacked microchannel plates (MCP). The sets of deflection plates are included to remove the interaction region from the line of sight of the ion detector. The metastable $2 s$ population is monitored by observing the Lyman- $\alpha$ radiation quenched in the same electric field used to collect the ions (the $2 s$ and $2 p$ states are fully coupled in an electric field of about $400 \mathrm{~V} / \mathrm{cm}$ ). Lyman- $\alpha$ radiation from the interaction region is collimated by a $\mathrm{MgF}_{2}$ lens and passes through a Lyman- $\alpha$ filter onto a MCP detector VUV sensitized with CsI.

The amplitude and the switch-on time of the external electric field can both be varied. This allows the effects of the external field to be distinguished from the effects of the collective field due to the ions and electrons. Each set of experimental measurements has been performed twice, once with the extraction field switched on during the laser pulse, and once with the extraction field off during the laser pulse, but switched on very rapidly (rise time about $13 \mathrm{~ns}$ ) immediately after the laser pulse.

The ion and Lyman- $\alpha$ signals are measured as a function of time and recorded as wave forms by a fast digitizing oscilloscope. The contents of the oscilloscope's memory are transferred to the PC for analysis at the end of each experimental run. Integrating over the wave forms provides the yield of a particular species at a specific laser intensity. Analysis of the time dependence of the signal provides important insight into the dynamics of the interaction both in the shape of the time-of-flight profile of the ions as well as the time of occurrence of the Lyman- $\alpha$ decay with respect to the laser flash. The wave forms are thus retained as an experimental record.

The ion and Lyman- $\alpha$ signals have been measured as a function of the laser intensity from 0.4 to $200 \mathrm{MW} / \mathrm{cm}^{2}$ (the maximum intensity available from the dye laser tuned to 243 $\mathrm{nm})$. The ion and Lyman- $\alpha$ signals have been recorded on consecutive experimental runs (of about 30 min duration). Each ion datum point is the average of 70 laser shots, with a maximum statistical uncertainty $<4 \%$ for the laser intensity and $<7 \%$ for the integrated analog ion signal. Each 
Lyman- $\alpha$ datum point is the average of 100 laser shots. Considerable care has been taken to minimize the amount of scattered laser light entering the Lyman- $\alpha$ detector, which is mounted directly above and in direct line of sight of the interaction region. Nevertheless, a small background signal arising from the laser flash could not be screened out completely. This has been measured by switching the hydrogen discharge source off and recording the signal from the Lyman- $\alpha$ detector over the full range of intensities for 100 laser shots. The average is then subtracted from the average signal taken with the discharge switched on to yield the true Lyman- $\alpha$ signal.

The total systematic uncertainty in the laser intensity data is $\pm 20 \%$ and is obtained by quadrature addition of the uncertainties of the energy, pulse length, and spot size. The conversion of the integrated experimental signals to occupation probabilities requires an accurate knowledge of atom densities as well as detector efficiencies. For this reason it is not possible to place the data on an absolute scale to an accuracy better than $\pm 80 \%$ in the case of the ion data and $\pm 120 \%$ in the case of the Lyman- $\alpha$ data.

\section{THEORETICAL MODEL}

The space-time behavior of ions produced in the multiphoton process is analyzed using a fluid dynamics approach based on the Boltzmann transport equations, while electrons are introduced in an averaged way according to Giammanco [13]. We assume radial symmetry around the axis of the laser beam, although this is strictly true only in the absence of the external field. This approximation greatly simplifies the numerical integration, and nevertheless allows us to reproduce the observed features very well. The basic set of equations is

$$
\begin{aligned}
\frac{\partial N_{M}(r, t)}{\partial t}= & \beta_{M} \frac{\left[I_{L}(r, t)\right]^{2}}{S(r, t)}\left[N_{G}-N_{M}(r, t)-N_{i}(r, t)\right] \\
& -\beta_{i} I_{L}(r, t) N_{M}(r, t)-K_{M}\left[E_{T}(r, t)\right]^{2} N_{M}(r, t),
\end{aligned}
$$

$$
\begin{aligned}
\frac{\partial N_{i}(r, t)}{\partial t}+\frac{1}{r} \frac{\partial\left[r \nu_{i}(r, t) N_{i}(r, t)\right]}{\partial r} & =\beta_{i} I_{L}(r, t) N_{M}(r, t), \\
\frac{\partial \nu_{i}(r, t)}{\partial t}+\nu_{i}(r, t) \frac{\partial \nu_{i}(r, t)}{\partial r}= & \frac{e}{M} E_{T}(r, t) \\
& -\frac{k T_{i}}{M} \frac{\partial \ln \left[N_{i}(r, t)\right]}{\partial r},
\end{aligned}
$$

where $N_{i}(r, t), N_{M}(r, t), \nu_{i}(r, t), I_{L}(r, t)$, and $N_{G}$ are the ion and metastable densities, ion fluid velocity, laser intensity, and deuterium beam density, respectively. $\beta_{M}$ $=\sigma_{M} /\left[\Gamma\left(h \nu_{L}\right)^{2}\right]$ and $\beta_{i}=\sigma_{i} / h \nu_{L}$ are the probabilities of metastable excitation and ionization, respectively, where $\sigma_{M}$ and $\sigma_{i}$ are the cross sections of metastable excitation and ionization, $\Gamma$ is the width of resonance, and $h \nu_{L}$ is the energy of a laser photon. $M, e$, and $T_{i}$ are the mass, charge, and temperature of the ions. $S(r, t)$ is the shift of $2 s$ resonance induced by the total electric field and is given by

$$
S(r, t)=\frac{\left\{K_{S}\left[E_{T}(r, t)\right]^{2}+\Delta \nu_{L}\right\}^{2}}{\Gamma^{2}}+1,
$$

where $K_{S}=3.6 \times 10^{8} \mathrm{~Hz} / E^{2}$ and $K_{M}=2.5 \times 10^{8} \mathrm{~Hz} / E^{2}$ are the coefficients of the static Stark shift and of level mixing, respectively [14] (the electric field is expressed in electrostatic units), and $\Delta \nu_{L}$ is a laser detuning factor, which we introduce to compensate for the external electric field when switched on before the laser pulse. The dynamic Stark shift is not expected to be significant within our range of laser intensities [15], and is therefore neglected in the present theoretical approach.

The collective field $E_{\rho}(r, t)$ is evaluated by solving the equation

$$
\frac{1}{r} \frac{\partial E_{\rho}(r, t)}{\partial r}=4 \pi e\left[N_{i}(r, t)-N_{e}(r, t)\right],
$$

where $N_{e}(r, t)$ is the electron density [see Eq. (7) below]. The collective field is set at zero at the center of mass of the charge distribution. Radial symmetry is broken in the presence of an external field $E_{0}$ directed along the axis of the time-of-flight (TOF) spectrometer. Taking the $x$ axis along the deuterium beam towards the TOF spectrometer, the $y$ axis in the direction of the Lyman- $\alpha$ detector, and the $z$ axis along the laser beam, an arbitrary position in a radial section through the laser beam and parallel to the $x y$ plane can be expressed in polar coordinates $(r, \theta)$, where the angle $\theta$ is measured from the positive $x$ axis. For an arbitrary position $r$ at time $t$ in a radial section, the total electric field is given by

$$
\vec{E}_{T}(r, t)=\left[E_{0}+E_{\rho}(r, t) \cos \theta, E_{\rho}(r, t) \sin \theta\right] .
$$

When the external field is present during the laser pulse, it may in part compensate the horizontal component of the collective field. Maximum compensation occurs along the negative $x$ axis $(\theta=\pi)$ in the direction opposite to the TOF entrance. In our experimental conditions, the electric field has a negligible effect in shifting the two-photon resonance, the main effect being related to the level mixing of the nearly degenerate $2 s-2 p$ manifold. With these assumptions, the ion production phase depends on the square of Eq. (6). Of course, radial symmetry is maintained during the production phase when the external field is switched on after the laser pulse.

During the time that the ions are moving towards the TOF entrance the initial shape of the ion profile produced by the laser pulse can be modified when the collective field $E_{\rho}$ reaches values comparable with the external field $E_{0}$. Along the $x$ axis the collective field accelerates the ions whose initial position is on or near the positive $x$ axis, and correspondingly decelerates the ions on the negative $x$ axis. One component of the ion flux is pushed ahead towards the TOF entrance, while the other component is repelled back and some of the ions may not reach the detector. The expansion of the ion cloud along the $y$ axis is driven by the $E_{\rho}$ field only and may result in an expansion to a size greater than the TOF entrance diameter. Both expansions can prevent a number of ions from reaching the detector. An observed saturation in the yield of ions may be caused primarily by the Coulomb explosion of the ion cloud, rather than a true satu- 
ration of the ion signal in the interaction volume. Not only the ion signal but also the quenching of metastable $2 s$ atoms is affected when $E_{\rho}$ reaches values comparable with $E_{0}$. This is demonstrated by the time dependence of the Lyman- $\alpha$ emission especially in the case where the external field is switched on after the laser pulse.

The space-time evolution of the electron density is evaluated according to a procedure described in detail in [6] and [13]. We assume an initial Gaussian radial profile. In the subsequent time evolution, the spatial profile retains its shape provided that the collective field is a linear function of radius. As demonstrated in [6], this approximation holds true within the half-width of the radial distribution. On the other hand, the main contribution to the electron field occurs just close to the half-width of the distribution. Hence, we represent the space-time evolution of electron density as

$$
N_{e}(r, t)=N_{G} g(t) f_{e}(t) \exp \left\{-f_{e}(t) \frac{\left[x_{s e}(t)-x\right]^{2}+y^{2}}{d^{2}}\right\},
$$

where $f_{e}(t)$ is related to the width of profile and $x_{s e}(t)$ represents the position of the center-of-mass of the electron charge distribution. $d$ is the half-width of the initial charge profile. $g(t)$ represents the degree of ionization at the maximum of the laser intensity radial profile, i.e., $g(t)$ is a solution of the rate equations (1) and (2), with $\nu_{i}$ set equal to zero. The problem is now reduced to finding the constitutive equations for the time evolution of the functions $f_{e}(t)$ and $x_{s e}(t)$. According to $[6,13]$, the time evolution in radial symmetry is given by a solution of the second-order total time derivative equations

$$
\begin{gathered}
D^{2} f_{e}(t)=\frac{3}{2} \frac{\left[D f_{e}(t)\right]^{2}}{f_{e}(t)}-2\left(\frac{f_{e}(t)}{\tau_{e}}\right)^{2} f_{e}(t)^{2 / 3} \\
+\omega_{p e}^{2} g(t) f_{e}(t)\left[f_{i}(t)-f_{e}(t)\right], \\
D^{2} x_{s e}(t)=-\frac{e}{m} E_{0}+\frac{1}{2} \omega_{p e}^{2} g(t) f_{i}(t)\left[x_{s i}(t)-x_{s e}(t)\right],
\end{gathered}
$$

where $\omega_{p e}=\left[\left(4 \pi e^{2} / m\right) N_{G}\right]^{1 / 2}$ is the electron plasma frequency, and $e$ and $m$ the electron charge and mass, respectively. The coefficient $\tau_{e}$ is the time constant related to the random motion of electrons, i.e., $\tau_{e}=\left(m / 2 E_{e}\right)^{1 / 2} d$, where $E_{e}$ is the initial energy of electrons immediately after multiphoton ionization, i.e., in our case $E_{e}=3 h \nu_{L}-I_{P}$ where $I_{P}$ is the ionization potential.

In the framework of the model described in [6,13], the ion evolution is described by a similar set of equations for $f_{i}(t)$ and $x_{s i}(t)$, with the assumption that the ions also have a Gaussian shaped profile with a normalized ion density $f_{i}(t)$ at the center-of-mass $x_{s i}(t)$ of the ion charge distribution. In the present case, these functions are evaluated from the numerical solution of Eqs. (1), (2), (3), and (5).

A stationary solution of Eq. (9) around the ion charge center of mass allows us to work out a condition for electron coupling. The stationary solution requires that electron oscillations occur in a time shorter than the laser pulse duration $\tau_{L}$, i.e., $\tau_{L} \gg 1 / \omega_{p e}$, which implies a charge density
$>10^{6}$ charges $/ \mathrm{cm}^{3}$. In this case, the spatial separation of the electron center of mass and the ion center of mass is given by

$$
\Delta x_{e i}=\frac{2 e}{m} \frac{E_{0}}{\omega_{p e}^{2} g(t) f_{i}(t)} .
$$

Strong coupling requires $\Delta x_{s e}<2 d$. Assuming a negligible ion expansion during the laser pulse, i.e., $f_{i}(t)=1$, in our experimental conditions $\left(E_{0}=1.7 \mathrm{esu}, d=0.01 \mathrm{~cm}\right.$, and $N_{G}$ $=5 \times 10^{11}$ atoms $/ \mathrm{cm}^{3}$ ), electron-ion coupling requires a degree of ionization $g(t)>10^{-2}$ that corresponds to a regime of laser intensity where the ion signal begins to saturate. However, in this charge density region, a Coulomb explosion of the ions occurs for times of the order of the laser pulse duration. Roughly speaking, the time constant of the ion expansion depends on the ion plasma frequency $\omega_{p i}$. The condition $\tau_{L} \gg 1 / \omega_{p i}$ is achieved for a charge density higher than $4 \times 10^{9}$ charges $/ \mathrm{cm}^{3}$, corresponding to a degree of ionization higher than $10^{-2}$. Therefore, a fast decrease of $f_{i}(t)$ leads to a weaker electron trapping. On the other hand, our experimental results show that electrons do in fact play a role only during the laser pulse.

The system of partial derivative equations is numerically integrated by using a modified LAX finite difference method with adaptive spatial step size, while the total time derivative equations for the electrons are integrated by a fourth-order Runge-Kutta method. Nonlinear terms of convective derivatives in Eqs. (2) and (3) are generally responsible for the growth of numerical instabilities in the presence of steep density gradients [16]. In fact, when a Coulomb explosion of the electron-ion cloud takes place, ions are pushed outward by the internal field and accumulate at the boundary of the cloud in a stepwise profile. The Coulomb explosion generates a collisionless shock wave.

In order to make the algorithm of our solution stable in the presence of a shock-wave-like evolution, each point $j$ of the spatial grid at an instant $t_{j}$ is first replaced by the average of values at the positions $j-1$ and $j+1$. This technique is still not sufficient to avoid instabilities at densities higher than $10^{10}$ charges $/ \mathrm{cm}^{3}$. The main source of instability arises from the terms $\nu_{i}(r, t) \partial N_{i}(r, t) / \partial r$ in the continuity equation [Eq. (2)] and $\nu_{i}(r, t) \partial \nu_{i}(r, t) / \partial r$ in the equation of motion [Eq. (3)]. The stability of the solution depends critically on the condition $(\Delta t / \Delta x) V_{\max } \leqslant 1$ where $V_{\max }$ is the maximum value of the velocity over the spatial grid, and $\Delta t$ and $\Delta x$ are the initially selected time and spatial steps of integration. Therefore, at each time step, the spatial step eventually increases until the stability condition is fully satisfied. As a consequence, the method we have selected to find solutions results in a smoothing of the density gradients and hence details of the radial ion profile are lost. On the other hand, the assumption of radial symmetry implies by itself a loss of information about the real ion distribution. Moreover, results of our model in the high-density regime show that the recorded ion time shape retains only a partial memory of the initial distribution as determined at the end of the multiphoton ionization process. The possible influence on the ion distribution of the spatial modulation of the electron profile, which occurs on the wings of the distribution where the electron plasma frequency changes noticeably as density decreases, is missing because of the assumption of self-similar 


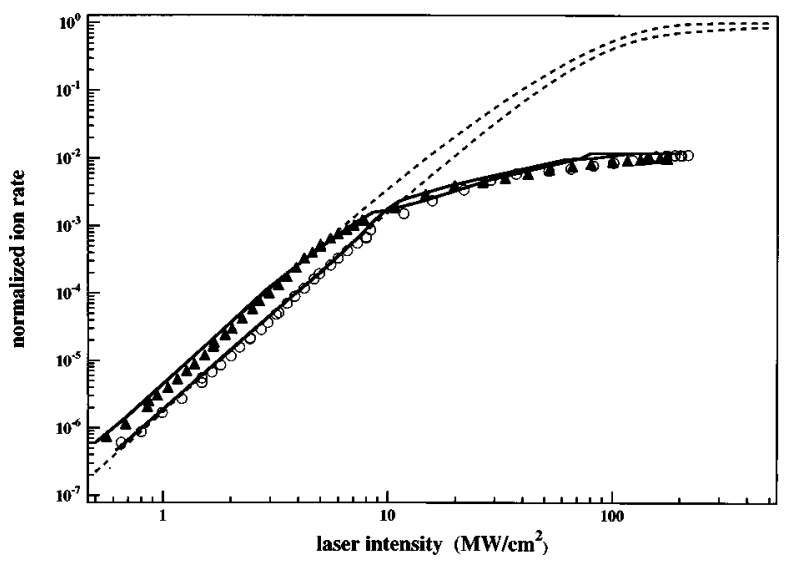

FIG. 2. Normalized ion rate vs laser intensity. The measured ion yields are normalized to the theory at $0.6 \mathrm{MW} / \mathrm{cm}^{2}$. Full triangles: external field switched on after the laser pulse. Open circles: external field switched on during the laser pulse. Dashed lines: ion rate from the rate equations (single-particle model). Solid lines: ion rate from the collective model.

evolution of the initial Gaussian profile [Eq. (7)]. Although our model is not an exact solution, it nevertheless gives a good quantitative as well as qualitative description of the observed dependences of the experimental signals on laser intensity.

We separately calculate the ion collection efficiency, which depends only on an expansion along the $y$ axis. In this case, the system of equations is solved with $E_{0}=0$ and $\theta$ $=0$ (see above). The efficiency is defined as the ratio between the ion flux crossing the TOF entrance aperture (and therefore reaching the detector) and the total ion flux integrated along the $y$ axis. The algorithm we have selected has the advantage of being flexible with regard to the conditions of integration; i.e., the solution is stable in the presence of spatial gradients of the external electric field and for the overall time interval of interest. Using this approach, we can exactly reproduce the experimental conditions along the axis of TOF spectrometer.

\section{RESULTS AND DISCUSSION}

In this section we present a series of measurements of the yields and time-of-flight spectra of ions and the time dependence of the quenched Lyman- $\alpha$ radiation produced in threephoton ionization of atomic deuterium. In each of the Figures 2-6 a comparison is made between the experimental results and the collective model.

Figure 2 shows the total measured ion rates for two cases, i.e., (i) with external field delayed until after the laser pulse and (ii) with the external field present during the laser pulse. For each case the experimental data are compared with a theoretical curve representing a simple rate equation model (single-particle model) and a theoretical curve calculated using the collective model. The experimental data are normalized to the theoretical curves at $0.6 \mathrm{MW} / \mathrm{cm}^{2}$. The experimental ion rates have been evaluated from the experimental signals using a best estimation of the overall collection efficiency. This procedure is affected by an estimated uncertainty of $\pm 80 \%$. However, the discrepancies for laser intensities higher than $10 \mathrm{MW} / \mathrm{cm}^{2}$ are so large that they can only be ascribed to the occurrence of a new physical effect not included in the simple rate equation (single-particle) model. The experimental ion rates correspond to the ionization degree averaged over the zone illuminated by the laser beam. Therefore in both theoretical models we have calculated the ion density profile over the interaction volume and hence we carry out the average degree of ionization at a selected laser intensity.

For laser intensities below $6 \mathrm{MW} / \mathrm{cm}^{2}$, the data sets fit to straight lines of slope $3.10 \pm 0.13$ (external field present during the laser pulse) and 3.09 \pm 0.17 (delayed external field), confirming that the ionization is third order as expected. In the case of the delayed field the ion yields are greater by a constant factor. This is expected since the presence of the external field during the laser pulse leads to a reduction in the lifetime of the intermediate metastable $2 s$ state with a corresponding decrease in the ionization probability. The experimental data show a reduction in ionization probability of $2.5 \pm 0.8$, which compares favorably with the factor of 2 difference in the theoretical curves.

At laser intensities above $6 \mathrm{MW} / \mathrm{cm}^{2}$, both sets of experimental data merge and the ion yields exhibit saturation. The convergence of the data at these laser intensities is consistent with the presence of significant collective fields in the interaction volume that swamp the influence of the external field. This is broadly confirmed by the theoretical results that illustrate that the inclusion of collective effects in this intensity regime leads to an earlier saturation (compared with the single-particle model) and brings the theory closer to experiment. The onset and the saturation of the ion yields in Fig. 2 (shape of the curves) are well described by the theory.

Before analyzing the ion time-of-flight shapes, let us discuss the main features of the Lyman- $\alpha$ emission, which is a suitable probe for collective effects. Figure 3(a) shows theoretical and Fig. 3(b) experimental data for the time dependence of the Lyman- $\alpha$ emission at four characteristic laser intensities and with a delayed external field. The collection field is switched on $40 \mathrm{~ns}$ after the laser pulse has ended. In Fig. 3(b) two peaks are visible (except at $12 \mathrm{MW} / \mathrm{cm}^{2}$ ). The early peak (occurring between 10 and $30 \mathrm{ns)} \mathrm{corresponds} \mathrm{to}$ Lyman- $\alpha$ emission during the laser pulse, and evidently arises from the transfer of population from the $2 s$ to the $2 p$ level, due to the presence of a collective electric field arising from the electrons and ions in the interaction volume. This interpretation is supported by the increasing dominance of this peak as the laser intensity increases. The second peak (occurring between 40 and $80 \mathrm{~ns}$ ) is due to the metastable atoms that have survived the laser interaction and are subsequently quenched in the external electric field. These experimental data illustrate the efficacy of using quenched Lyman- $\alpha$ emission from the nearly degenerate $2 s-2 p$ states as an experimental monitor of the onset of relatively small collective fields in the interaction region. Our data therefore provide direct evidence of the presence of collective fields induced by the plasma.

Figure 3(a) illustrates the theoretical modeling of the time dependence of the Lyman- $\alpha$ signal. The thick lines are calculated including only ions while the thin lines account for the electron influence in the presence of electrons as described in the theoretical section. The electric field is switched on after the end of the laser pulse with a rise time 


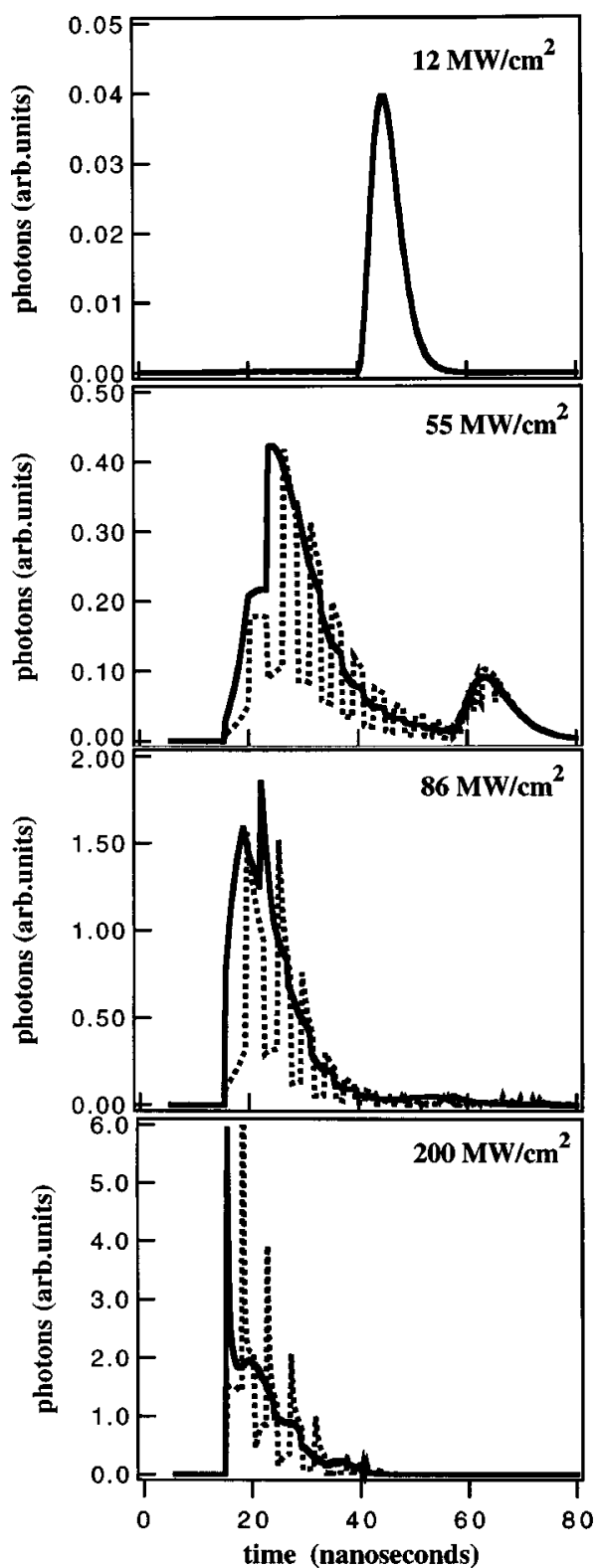

(a)

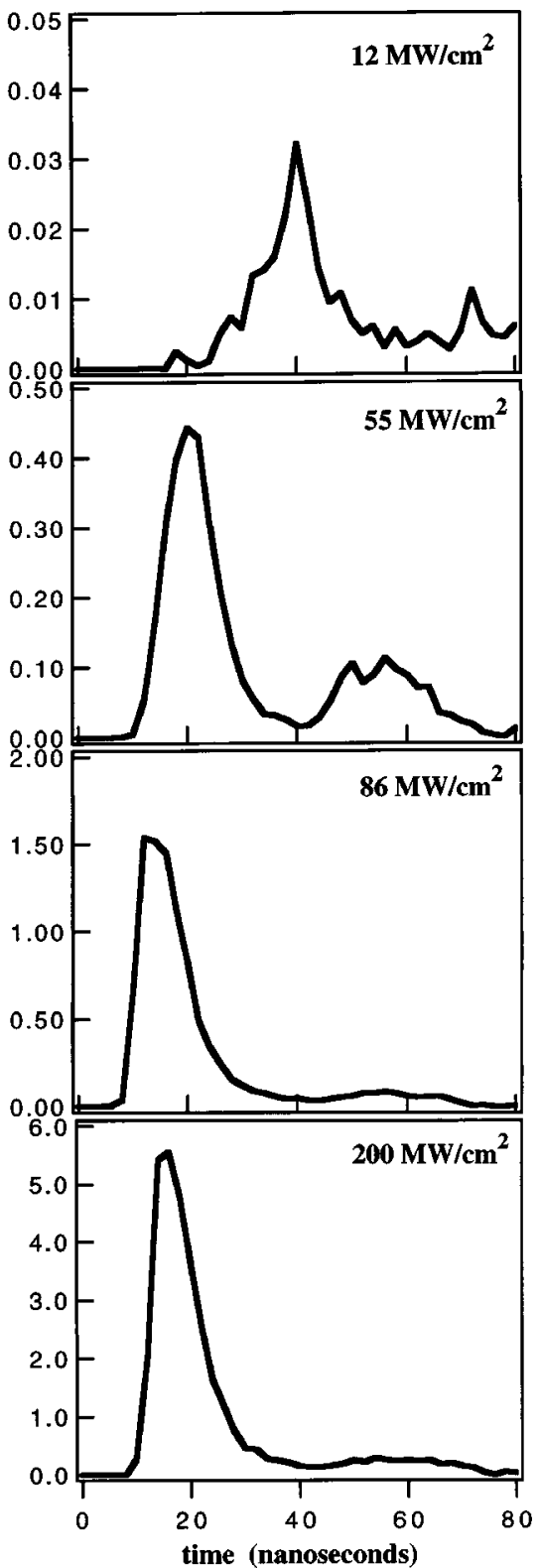

(b)

FIG. 3. Lyman- $\alpha$ signal as a function of time for different laser intensities with the external field switched on after the laser pulse. (a) Calculated profiles from the collective model. Solid lines: ions only; dashed lines: electrons included. (b) Experimental profiles.

of $13 \mathrm{~ns}$ as in the experiment. The rise time of the second peak depends on the rise time of the external field, while the fall time is related to the lifetime of the atomic level. The broad features of the experimental data are well produced. Notice that in both experiment and theory the maximum in the yield of Lyman- $\alpha$ radiation observed during the laser pulse shifts to earlier times as the laser intensity is increased.

The theoretical data in the presence of electrons do, however, show a marked oscillatory structure in the early peaks. This structure is due to the oscillation of the width of the electron profile around the ion one. Since ion expansion is negligible, the frequency of oscillation is related directly to the electron plasma frequency according to Eq. (8) $\left[f_{i}(t)\right.$ $=1]$. The timing resolution of our experiment was not sufficient to observe these oscillations and we cannot ascertain directly a possible role of electrons.
Figure 4 shows similar results as Fig. 3, except in this case the external field is present during as well as after the laser pulse. In this case, the later Lyman- $\alpha$ peak is almost absent in all cases, indicating that almost no metastables have survived beyond the end of the laser pulse. In the case of the external field switched on during the laser pulse, the collective field acts in a different way with respect to the center of mass of the ion distribution where the collective field is zero as previously remarked. Assuming radial symmetry along the axis of detection, the collective field adds to the external field towards the entrance of the TOF spectrometer and subtracts in the opposite direction. Therefore, in one section of the ionized zone within the interaction region the external field can be partially or totally compensated for by the collective field as the density increases. Taking account of the fact that during the laser pulse ions move towards the 


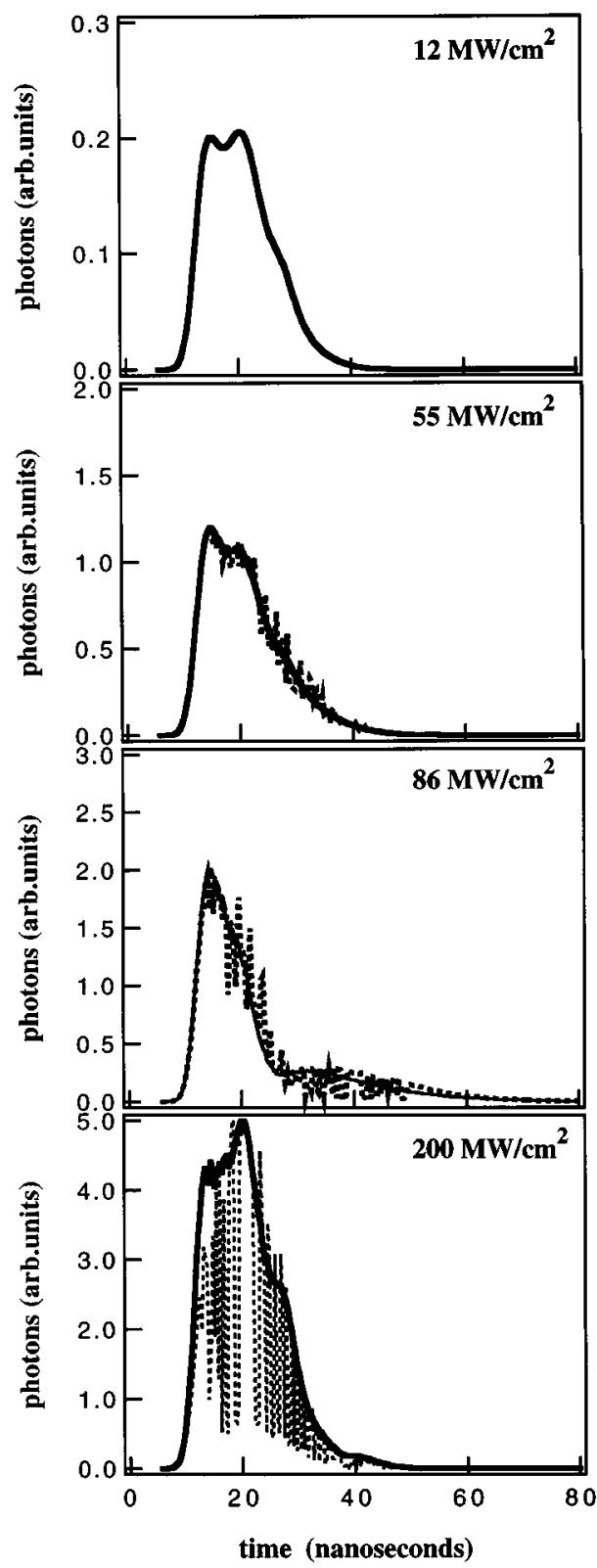

(a)

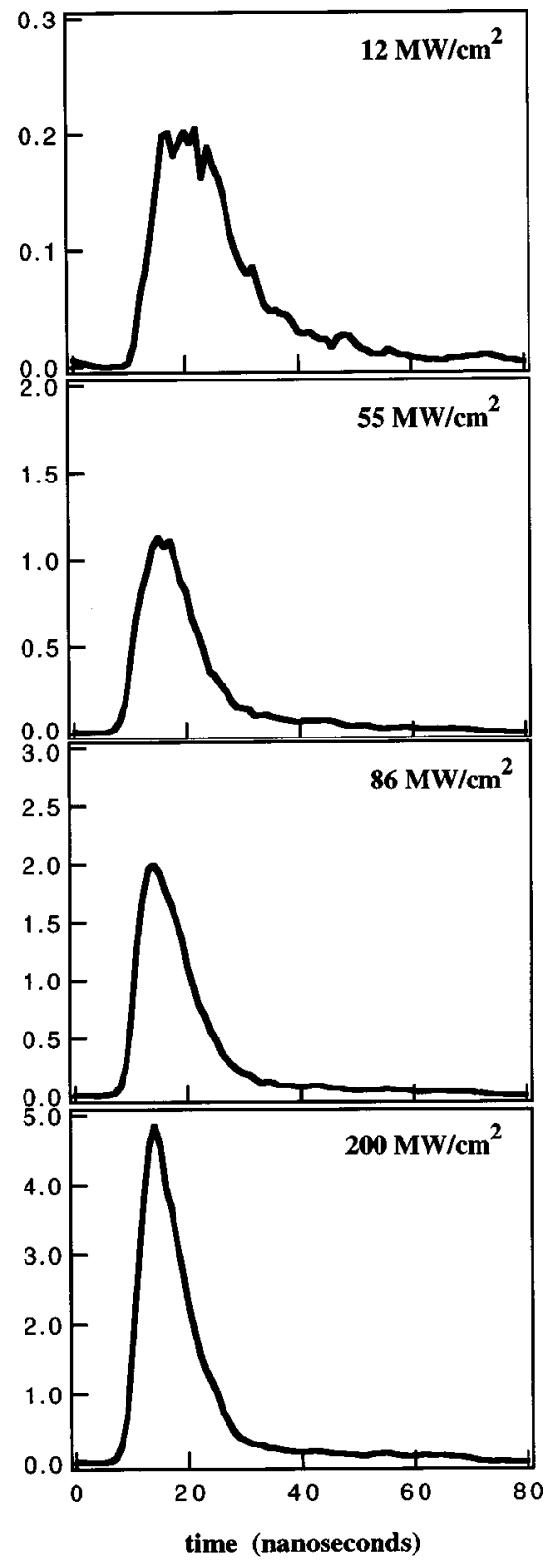

(b)

FIG. 4. Lyman- $\alpha$ signal as a function of time for different laser intensities with the external field switched on during the laser pulse. (a) Calculated profiles from the collective model. Solid lines: ions only; dashed lines: electrons included. (b) Experimental profiles.

entrance aperture of the TOF spectrometer, the compensated zone can fall at some instants close to the maximum of the laser intensity spatial profile, leading to an enhancement of the metastable production and, hence, to a decrease of Lyman- $\alpha$ emission. Therefore, we can expect a narrowing of the emission peak during the laser pulse, which is then followed by a tail extending beyond the end of laser pulse. Compensation disappears as the density increases and the collective field overcomes the external field, but this occurs far beyond the upper charge density limit reached in our experiment. The oscillatory structure is in this case due to the oscillation of the electron center of mass around the ion center of mass and is related to the electron plasma frequency weighted by the ion width according to Eq. (9). In this case, at higher laser intensity, neither the motion of the ions during the laser pulse, nor the enhancement of the ionization due to the collective field can be neglected. It turns out that the frequency of oscillation can be higher than in the previous case $\left[f_{i}(t)>1\right]$.

The results of Figs. 3 and 4 confirm the importance of the observation of Lyman- $\alpha$ emission as a diagnostic tool in the control yields of laser-deuterium interactions. We can confidently conclude on the basis of all of these data that the ion TOF spectrum is largely dominated by the internal Coulomb repulsion of the ion cloud during the collection phase. In these circumstances, it is clear that spatial details of the ionization process are not observable.

Figure 5 shows the time-of-flight spectra of the ions at laser intensities higher than $6 \mathrm{MW} / \mathrm{cm}^{2}$, where the ion rate starts to deviate from the rate-equation curve (see Fig. 2). As above, Fig. 5(a) refers to the theoretical curves while Fig. 5(b) shows the experimental time-of-flight profiles. We have 

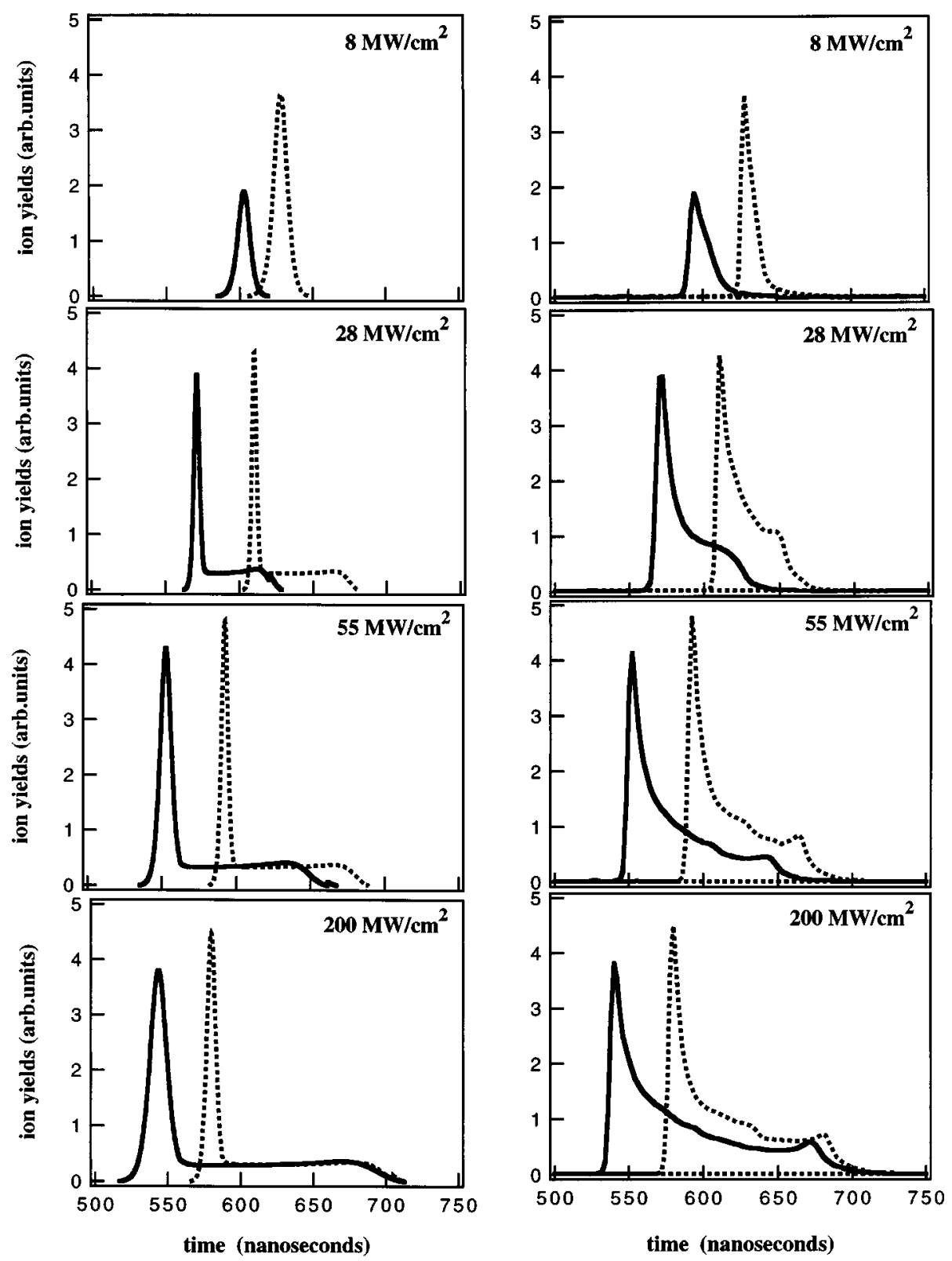

(a)

(b)

FIG. 5. Ion time-of-flight spectra for different laser intensities. Solid lines: external field switched on during the laser pulse. Dashed lines: external field switched on after the laser pulse. (a) Calculated profiles from the collective model. (b) Experimental profiles.

selected the same vertical scale in both diagrams in order to facilitate the comparison between theory and experiment.

The theoretical ion profiles have been calculated in the absence of electrons, because the electrons do not change the general features of the profiles. The theoretical profiles are in good agreement with the measured TOF spectra with regard to both peak heights and the extension of the tail. The difference between theory and experiment regarding the shapes of the tails is not surprising considering the inherent limitations of a one-dimensional modeling of the ion cloud, but a second factor may be the algorithm used in the integration. The transition from the peak to the tail is less marked when the adaptive step size in the integration is excluded, at least before the numerical instability takes place. As pointed out above, we have selected an integration method that smooths the density gradients in order to improve stability. As ions accumulate into the first peak (a sort of shock-wave front) leading to a growth of density gradient, the spatial step increases to ensure that the stability condition is satisfied. This increases the relative weight of the spatial zone where the density gradient is smoother. We believe that a different choice of integration method (which would lead to a considerable increase in computational times) would not fully account for the recorded ion time profiles as long as the onedimensional approach is maintained. As pointed out in Sec. III (theoretical model), radial symmetry of the laser profile is broken when collective effects take place.

Let us first consider the case where the external field is applied after the end of laser pulse. The maximum ion density occurs in the radial section through the laser beam coinciding with the TOF axis. As the radial section moves along the laser beam away from the TOF axis the ion density de- 
creases. We can image the ion cloud as formed by a series of radial sections, parallel to each other and to the TOF axis, with a one-dimensional ion density profile and an average ion density progressively decreasing off-axis. Apart from mutual interactions, the time evolution of each axial section depends on the local ion density, i.e., as the density decreases, the arrival time of the first peak and the width of the tail converge to their unperturbed values. These radial sections contribute to an increase in the ion signal just after the main peak and before the end of tail. This simple physical picture holds true also in the case of the external field switched on during the laser pulse. In fact, the features of the time evolution depend mainly on the average ion density.

Figure 5(b) shows that the ion profiles have similar shapes in both cases. It demonstrates that the TOF spectra are largely dominated by the Coulomb explosion and that the details of the photoionization process are partially or totally missing. However, the detected TOF shape retains some memory of the shape of the ionized volume. Figure 5(b) shows that the ion peaks and overall shapes, recorded when the external field is switched off during the laser pulse, are narrower than that in the case of the field switched on. Considering that the average ion density is higher at the same laser intensity, this fact seems in contrast with a TOF shape dominated by the Coulomb explosion. Nevertheless, we have to take into account that the expansion depends on the shape of the ion cloud at the end of the ionization process. When the external field is switched on, the value of the external field is high enough to accelerate ions, produced at some instants, through the laser radial profile. The combination of collective and external fields induces an additional asymmetry of the ionization probability (see above), it turns the ion profile spread out just during the production phase whereas it does not occur when the external field is switched off. Hence, the further evolution retains a sort of averaged memory of the initial spatial shape.

The dependence of the TOF spectra on laser intensity allows us to obtain information about the role played by the electrons at high laser intensity. Electron trapping screens the external field and delays the collection of ions. This leads to an increase of the ion arrival time, while we observe consistently a decrease in the time of flight as the charge density increases. It turns out, however, that electrons are collected quickly and within our range of investigation, at a time close to the end of the laser pulse and also in the case of the delayed field. Although the electrons do not affect the ion collection, electron oscillations around the ion cloud during the laser pulse can modify the shape of the total collective field through a partial screening of the ion field. In this case, Lyman- $\alpha$ emission should be modulated at a frequency of the order of the electron plasma frequency. Our setup does not allow us to detect a possible modulation of photon emission because the signal must be averaged over a large number of laser pulses. However, it is our intention to improve the photon detection efficiency in order to get additional information on charge density.

Finally, we remark that saturation depends only minimally on a reduced collection efficiency at the TOF entrance. When Coulomb explosion occurs the collection efficiency drops asymptotically from 1 to about 0.3 at the maximum laser intensity within our range. Figure 6 shows a compari-

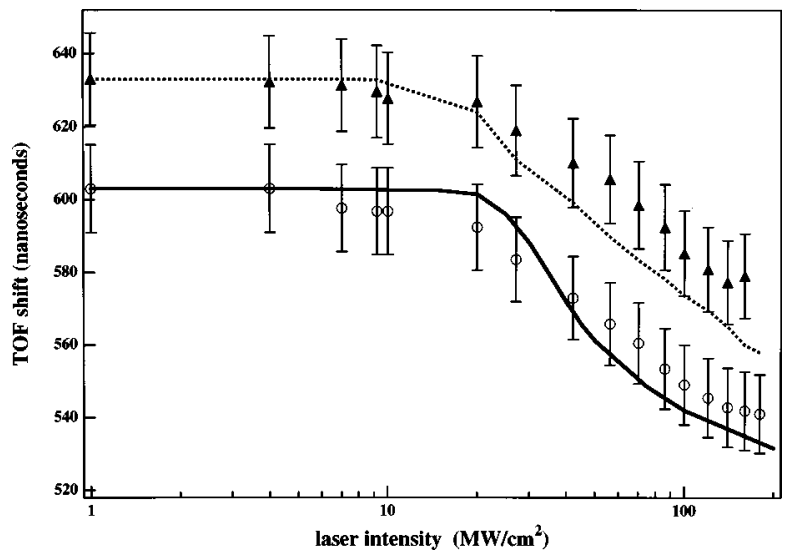

FIG. 6. Arrival time of first ion peak vs laser intensity. External field switched on after the laser pulse: full triangles: experiment; dashed line: collective model. External field switched on during the laser pulse: open circles: experiment; solid line: collective model.

son between measured and calculated arrival time of ion peak versus laser intensity. We obtain similar behavior for the measured TOF of the center of mass of the ions. As the laser intensity increases the center of mass of the recorded ion profile undergoes a shift towards earlier arrival time, which is represented in both cases by curves like those of Fig. 6. These results emphasize the role of the Coulomb explosion. Coulomb repulsion among ions acts as an internal force of the ion cloud. The center-of-mass acceleration is only due to the external field. As pointed out above, a fraction of the ions are pushed away from the TOF entrance and hence it is likely that only a few ions can find the TOF entrance again and with a much later arrival time. Therefore missing ions contribute to a growth of an apparent center-ofmass high-energy shift of the detected signal.

\section{CONCLUSION}

We have demonstrated the relevance of collective effects in a multiphoton experiment on deuterium. Deuterium has proved to be particularly suitable for the study of collective effects. It is one of the lightest atoms and therefore when ionized very susceptible to collective fields. Measurements of the total ion yield and the ion time-of-flight profiles as a function of laser intensity show dramatic effects as a result of the collective effect. The anomalous saturation of the ion yield has been observed. The ion time-of-flight profiles display a broadening and a shift of the leading edge of the profile to earlier times. The $2 s$ state is the resonant state in the multiphoton ionization process and is very susceptible to Stark mixing with the $2 p$ state in the presence of electric fields. From careful measurements of the time dependence of the Lyman- $\alpha$ emission, the existence of collective fields of the same order of magnitude as the extraction fields has been demonstrated. Detection of both the ion signal and the Lyman- $\alpha$ emission has provided us with two complementary diagnostic techniques for the study of collective effects.

We have developed a theoretical model based on a fluid dynamics description of the motion of the electrons and the ions produced in the multiphoton ionization process. In order to make the numerical calculations manageable, radial symmetry around the axis of the laser beam had to be assumed. 
Despite this approximation, we have been able to reproduce the essential features of the collective interactions very well. All essential features in the measured ion yield, the ion timeof-flight profiles and in the time-dependent yield of Lyman- $\alpha$ photons have been reproduced by the theoretical model. The model accounts for the overall nonlinear behavior of the central part of the Gaussian profile in the initial ion and electron distributions. The numerical solutions provide an important guide towards a complete description of non-neutral plasmas with strong density gradients.

\section{ACKNOWLEDGMENTS}

This work has been supported under the Scientific Research Program of the Irish Research Agency Forbairt and under the TMR program of the European Union. One of the authors (R.W.O'N.) gratefully acknowledges the support of the European Union Human Capital and Mobility program. The authors also wish to acknowledge Niall Murphy for his contributions to the mechanical design of the apparatus and to Bill Lanigan for his contributions to the electronics and the computer interfacing of the apparatus.
[1] P. Lambropoulos and S. J. Smith, Multiphoton Processes (Springer-Verlag, Berlin, 1984).

[2] J. S. Bakos, Phys. Rep. 31, 209 (1977).

[3] P. Zoller, J. Phys. B 15, 2911 (1982).

[4] K. Burnett, V. C. Reed, and P. L. Night, J. Phys. B 26, 561 (1993).

[5] F. Giammanco, Phys. Rev. A 40, 5160 (1989).

[6] F. Giammanco, Phys. Rev. A 40, 5171 (1989).

[7] E. Arimondo, C. Buckhardt, L. Qin, F. Giammanco, and A. Vellante, Opt. Commun. 71, 52 (1989).

[8] F. Giammanco and E. Arimondo, Europhys. Lett. 11, 31 (1990).

[9] T. Mossberg, A. Flusberg, and S. R. Hartmann, Opt. Commun. 25, 121 (1978).

[10] K. Miyazaki, T. Sato, and H. Kashiwagi, Phys. Rev. Lett. 43, 1154 (1979).
[11] K. Miyazaki, T. Sato, and H. Kashiwagi, Phys. Rev. A 23, 1358 (1981).

[12] L. Marmet, K. Hakuta, and B. P. Stoicheff, J. Opt. Soc. Am. B 9, 1038 (1992).

[13] F. Giammanco, in Plasma Collective Effects in Atomic Physics, edited by F. Giammanco and N. Spinelli (Edizioni ETS, Pisa, Italy, 1996), pp. 1-23.

[14] F. Schmidt-Kaler, D. Leibfried, S. Seel, C. Zimmermann, W. König, M. Weitz, and T. W. Hänsch, Phys. Rev. A 51, 2789 (1992).

[15] C. R. Holt, M. G. Raymer, and W. P. Reinhardt, Phys. Rev. A 27, 2971 (1983).

[16] W. H. Press, B. P. Flannery, S. A. Teukolsky, and W. T. Vetterling, Numerical Recipes (Cambridge University Press, Cambridge, 1986). 